Word count:

Abstract: 251 words

Article Body: 6677 words

Introduction: 636 words

Discussion: 1716 words

Tables: 4

Figures: 3

\title{
Reduced emotional responsiveness in individuals with marginal elevation in blood pressure within the normal range: Evidence from altered affect- modulated startle response
}

MEENAKSHI SHUKLA ${ }^{\text {a\#}}$, JENNIFER Y. F. LAU ${ }^{\mathrm{a}}$, SHMUEL LISSEK ${ }^{\mathrm{b}}$, RAKESH PANDEY $^{\mathrm{c}^{*}} \&$ VEENA KUMARI ${ }^{\mathrm{d}^{*}}$

*Joint senior author

a Department of Psychology, Institute of Psychiatry, Psychology, \& Neuroscience, King's College London, London, UK

\#Current address: Department of Psychology, Magadh University, Bodh Gaya, India

${ }^{b}$ Department of Psychology, University of Minnesota Twin Cities, Minneapolis, USA

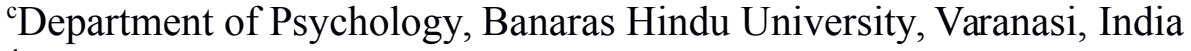

${ }^{\mathrm{d} C e n t r e}$ for Cognitive Neuroscience, College of Health and Life Sciences, Brunel University

London, Uxbridge, UK

*Correspondence to:

Rakesh Pandey, $\mathrm{PhD}$

Department of Psychology

Banaras Hindu University

India

E-mail: rpan_in@yahoo.com

Phone: + 91 (0)542-2369967

Financial Support: This research was supported by a Commonwealth Split Site Scholarship Award to Meenakshi Shukla (Ref: INCN-2016-232) and is based on part of her Ph.D. research data collected under the said award. 


\section{Highlights}

- Affective startle modulation was used to examine emotional reactivity associated with BP elevation in the normal range.

- Findings show that even marginal elevations in BP in the normal range may be associated with reduced emotional reactivity.

- Emotional dampening hypothesis of elevated BP and hypertension extends to include reduced involuntary emotional reactivity. 


\begin{abstract}
Reduced responsiveness to emotional stimuli ('emotional dampening') has been observed in normotensives with elevated blood pressure (BP) and hypertensives but it is not known whether this is due to aberrant responding to emotional information at the involuntary level and whether it is also associated with minimal elevations in BP in the normal range. In this study, we examined emotional dampening using the affect-modulated startle paradigm given its proven sensitivity to motivational states of approach and withdrawal, typically independent of conscious intentional control. Acoustically elicited startle eye-blink modulation was measured using electromyography of the orbicularis oculi muscle beneath the left eye in 59 healthy individuals while they viewed pleasant, unpleasant and neutral standardized pictures. The expected startle attenuation to pleasant pictures, and startle potentiation to unpleasant pictures, relative to neutral pictures, was found in people in the comparison ( $\mathrm{N}=29)$ but not elevated $\mathrm{BP}(\mathrm{N}=30)$ group. This finding was further supported by significant moderating effect (assessed using ANCOVA and sub-sample analysis) of BP on valence-startle amplitude relationship. The comparison BP group also showed slower latencies to response onset for pleasant stimuli compared to neutral and unpleasant, with no effect of valence in the elevated BP group. However, BP did not moderate the valence-onset latency relationship. Our findings indicate that previously reported emotional dampening associated with elevated $\mathrm{BP}$ extends to reduced involuntary emotional reactivity and to individuals with even minimal BP elevations (i.e. higher but still within the normal range). Future research needs to confirm these findings in hypertensive individuals, preferably using within-subjects designs.
\end{abstract}

Key words: Emotional responding, startle amplitude, elevated blood pressure, hypertension, BP 


\section{Introduction}

Hypertension is a health condition associated with a wide range of morbidity (Forouzanfar, et al., 2017). Elevation in blood pressure (BP) within the normal (Pei et al., 2011) as well as hypertensive range is associated with risk of cardiovascular diseases (Rapsomaniki et al., 2014) that alone account for two-thirds of the mortality rates (Mayor, 2016). Reduced responsiveness to affect-laden stimuli (termed 'emotional dampening'), irrespective of valence, has been reported in people with elevated BP within the normal range in the form of negative correlation between BP and emotion recognition (Pury, McCubbin, Helfer, \& Galloway, 2004; McCubbin, et al., 2011; McCubbin, et al., 2014; Shukla, Pandey, \& Lau, 2019), as well as in the hypertensive range compared to those with normal BPs (Shukla, Pandey, Jain, \& Lau, 2018; Shukla, Gupta \& Pandey, 2017). However, the conceptualisation of the phenomenon of reduced emotional responsiveness in these studies is limited in that they have focussed only on the subjective self-report and/or emotion recognition aspect of emotional responsiveness (e.g., reduced emotion recognition accuracy in affect-laden scenes, facial expressions of emotions, and written narratives) in individuals with elevated BP and hypertension; these studies did not examine sensitivity or reactivity to emotional stimuli. Moreover, it is not yet known whether this blunted affective response in relation to hypertension, or elevated BP, emerges at a relatively involuntary level of emotional reactivity. The present study aimed to address this gap and is perhaps the first to go beyond subjective self-report and emotion recognition paradigms to examine emotional reactivity, in relation to $\mathrm{BP}$ elevation within the normal range, using an established psychophysiological paradigm, namely, affective modulation of startle response (Vrana, Spence, \& Lang, 1988).

The simple startle reflex is known to be modulated by the concomitant presentation of emotionally-toned stimuli (e.g. pleasant or unpleasant pictures, sound or smells); if stimuli 
are pleasant, the reflex is attenuated, and if unpleasant, it is potentiated (Vrana Spence, \& Lang, 1988). This effect, known as affective startle modulation (ASM), is considered to index appetitive and aversive emotional states, resulting from the match (in case of unpleasant stimuli) or mismatch (pleasant stimuli) between the valence of the foreground stimulus and that of the startle probe (usually an aversive stimulus). This paradigm has been found to be useful in assessing automatic emotional reactivity across a range of emotional disorders (Grillon \& Baas, 2003). For instance, people with anxiety disorders show enhanced startle potentiation to unpleasant stimuli (Ray et al., 2009) while those with depressive disorders (Kaviani et al., 2004; Dichter \& Tomarken, 2008), comorbid anxiety and depression (TaylorClift, Morris, Rottenberg, \& Kovacs, 2011) or chronic stress (Lang \& McTeague, 2009) show reduced startle modulation to both pleasant and unpleasant stimuli.

The present study utilised, for the first time to our knowledge, affective modulation of the acoustic startle response to examine emotional reactivity in relation to $\mathrm{BP}$ elevation in the normal range compared to individuals with lower BPs (also within the normal range). It was hypothesized that elevated BP would be associated with attenuated ASM, given previous findings of reduced emotional responding to stimuli of positive as well as negative valence (Pury, et al., 2004; McCubbin, et al., 2011; McCubbin, et al., 2014; Jain, Shukla, \& Pandey, 2017) and a high prevalence of depression and chronic stress in people with elevated BP (AlKhathami, et al., 2017; Markovitz, Matthews, Whooley, Lewis, \& Greenlund, 2004) that are found to be associated with reduced modulation of startle reflexes to both pleasant and unpleasant stimuli. As in previous studies examining individual differences in emotional reactivity using the affect modulated startle paradigm (e.g. Corr, et al., 1995; Kumari, et al., 1996; Corr \& Kumari, 2013), we utilized startle amplitude (indicating the strength of startle response) as the primary measure for hypothesis testing but also explored the latencies to response onset and peak in association with elevated BP. 
Method

Participants

An a priori power analysis (using $G$ power 3.1.9.4) with an alpha value of 0.05 , a medium effect size of 0.25 (as recommended by Cohen (1969) for ANOVA), a power of 0.95, number of groups (between-subjects factor) equal to 2, number of repeated measurements equal to 3, and a moderate correlation of 0.40 among repeated measures for Repeatedmeasures ANOVA (including Valence $\mathbf{X}$ BP Group interaction) revealed that a total sample size of 52 would be sufficient to achieve the said power. We thus aimed to recruit relatively more participants compared to the required number (around 50\% more than that suggested by the power analysis) in order to arrive at a sample size equal to or greater than the required number (as suggested by the power analysis). Accordingly, participant recruitment was started through advertisement. In response to this advertisement 71 healthy participants (18-60 years; 47 women) from the general population turned up for the experiment. They were screened for any current or past diagnosis of a mental disorder, alcoholism and/or smoking, any physical disease condition such as thyroid, kidney or heart conditions, impaired vision or hearing, any regular medical prescription, pregnancy or lactation (9 potential participants failed screening and were excluded). Data from three more participants were excluded for different reasons (one left the experiment mid-way, and two were distracted by their mobile phones). The remaining 59 participants were assigned to comparison BP and elevated BP groups (see Data Analysis). Since hypertension is associated with enhanced negative emotions (Jonas \& Lando, 2000), Positive and Negative Affect Schedule (PANAS; Watson, Clark, \& Tellegen, 1988) was used to assess possible differences in positive and negative affectivity across the two groups prior to the startle experiment. The average $( \pm \mathrm{SE})$ resting SBP and DBP values along with age, gender distribution, and positive 
and negative affectivity for the two groups are presented in Table 1. However, for examining the moderating effect of BP (a continuous variable), data from all the participants $(\mathrm{N}=59)$ was used without categorizing them in any group (See Study Design and Data Analysis). The descriptive statistics, thus, for the all the participants are also presented in Table 1. Participants provided written informed consent to their participation and were compensated $£ 10$ for their time and, in addition, travel expenses.

Table 1: Overall and group-wise demographic information of the participants

\begin{tabular}{lccc}
\hline & & \multicolumn{2}{c}{ BP Groups } \\
Measure & $\begin{array}{c}\text { Overall sample } \\
(\mathrm{N}=59)\end{array}$ & $\begin{array}{c}\text { Comparison BP } \\
\text { group } \\
(\mathrm{N}=29)\end{array}$ & $\begin{array}{c}\text { Elevated BP group } \\
(\mathrm{N}=30)\end{array}$ \\
\hline Age range (years) & $19-54$ & $19-43$ & $19-54$ \\
Mean Age (SE) & $27.97( \pm 3.64)$ & $25.62( \pm 1.20)$ & $30.14( \pm 1.71)$ \\
Gender & 47 Females, & 25 Females, & 22 Females, \\
Mean SBP (SE) (mmHg) & 12 Males & 4 Males & 8 Males \\
Mean DBP (SE) (mmHg) & $106.03( \pm 0.82)$ & $103.62( \pm 1.09)$ & $108.15( \pm 1.07)$ \\
Mean Positive affectivity (SE) & $68.59( \pm 0.65)$ & $64.51( \pm 0.48)$ & $72.31( \pm 0.56)$ \\
Mean Negative affectivity (SE) & $27.05( \pm 1.14)$ & $26.07( \pm 1.45)$ & $28.00( \pm 1.74)$ \\
\hline
\end{tabular}

Fifteen participants described their ethnicity as Asian, 29 as Caucasian, 2 as African, 2 as Persian/Iranian/Turkish, and 7 as Mixed ethnicity. Four participants refused to state their ethnicity.

Study Design

A mixed factorial repeated-measures design was used with dichotomous BP groups as a between-subjects factor and valence (pleasant, neutral, unpleasant) as repeated-measures factor. Both systolic blood pressure (SBP) and diastolic blood pressure (DBP) data were collected at three time points immediately prior to beginning the study (see Procedure). To 
incorporate SBP and DBP into one factor, BP was modeled as a latent factor comprising of both SBP and DBP using structural equation modeling approach, which was then subjected to median split to create comparison BP and elevated BP groups. Latent blood pressure is a continuous variable and dichotomising continuous variable may lead to obtaining false positive results (Altman \& Royston, 2006). Therefore, to further support the findings of the aforesaid design that is fraught with the danger of dichotomizing the continuous variable (latent BP), the moderating effect of the continuous BP measure on the relationship of Valence and startle response was also assessed using one-way repeated measure analysis of covariance (ANCOVA) design (with latent BP as covariate and Valance as repeated measure). To further explore the moderating effect of latent BP in the relationship of valence and startle response, a sub-sample (or subgroup) approach was also used. The relationship of latent BP with different parameters of startle response for each of the three valence categories was compared across the two BP groups (viz., comparison and elevated BP group).

\section{Tools and Measures}

\section{The Positive and Negative Affect Schedule (PANAS)}

PANAS was developed by Watson, Clark, and Tellegen in 1988 as a self-report measure to assess the level of positive and negative affect of the respondents during a specified point of time or period. This affect schedule consists of $20 \operatorname{mood}$ states (10 positive and 10 negative) and the respondents are asked to use a 5- point Likert scale ranging from 1 (very slightly or not at all) to 5 (extremely) to indicate the extent to which they experience the given mood states during a specified time frame. The time frame of interest is indicated simply by varying the temporal instructions that ask respondents to indicate their affect "right now (that is, at the present moment)", "today", "during the past few days", "during the past week", "during the past few weeks", "during the past year", and "in general, that is, on the 
average". Since a person's current mood state could very likely influence their experience of emotions (in presented photographs), the moment instructions version of PANAS was used to assess the affective state of the participants just before the administration of the affect-startle task. The moment instructions version of PANAS has high internal consistency reliability (coefficient alpha for $\mathrm{PA}=.89$, and $\mathrm{NA}=.85)$ and satisfactory test-retest reliability (8-weeks retest reliability coefficient for $\mathrm{PA}=.54$, and $\mathrm{NA}=.45$ ). The scale also has good factorial and external validities (Watson, Clark, \& Tellegen, 1988).

\section{The Affect Startle Task}

The picture set and the design of the experiment was similar to that used by Giakoumaki et al. (2010). Participants were shown 54 images taken from the International Affective Picture System (IAPS; Lang, Bradley, \& Cuthbert, 2005). These comprised of 18 pleasant pictures (nos. 1610, 1750, 2070, 2080, 2550, 4614, 4650, 5830, 7200, 7280, 7330, $8030,8080,8120$ with $2040,2050,4520,4532$ for female participants only and 2030, 4210, 4180, 4232 for male participants only), 18 unpleasant pictures (nos. 1030, 1070, 1090, 1111, $2120,3000,3010,3030,3064,3100,3130,3140,3150,3210,6242,6570,9050$, and 9405), and 18 neutral pictures (nos. 2200, 5500, 5510, 7000, 7002, 7009, 7010, 7020, 7040, 7050, $7060,7080,7090,7100,7150,7170,7175$, and 7500 ). The arousal ratings (mean \pm SD) were $5.5 \pm 1.3$ for pleasant pictures for females, $5.1 \pm 0.8$ for pleasant pictures for males, $6.2 \pm 0.7$ for unpleasant pictures and $2.7 \pm 0.5$ for neutral pictures. Each of these pictures remained on the screen for $6 \mathrm{sec}$ and was followed by a varying inter-stimulus interval (blank screen) of 10-20 sec. In total, 48 startle probes $(50-\mathrm{ms}$ bursts of $105 \mathrm{~dB}$ white noise with instantaneous rise time) were delivered during the experiment. Of these, 36 probes were presented during the presentation of IAPS pictures (12/18 per valence category to diminish the predictability of probes) and 12 during the inter-stimulus intervals (to index baseline startle activity), at random points between $2-5 \mathrm{sec}$ after the onset of the images or inter-stimulus intervals. In 
order to ensure similar habituation effect on the startle responses to stimuli of all the three valence categories, the pictures were presented to all participants in the same pseudo-random order, similar to other studies examining affective startle modulation in humans (e.g., Giakoumaki, et al., 2010).

\section{Software and equipment}

The affective pictures, as well as the acoustic probes, were presented using E-prime 3.0 software (Psychology Software Tools, Pittsburgh, PA). The startle probe was presented to the participants through headphones (Philips SHL3160PP). The physiological recordings in the study and the processing of the EMG signals were done using Psylab 8 (Contact Precision Instruments, London, UK). For capturing the eyeblink startle response (EMG signals) three $4 \mathrm{~mm} \mathrm{Ag}-\mathrm{AgCl}$ electrodes were used and Signa Gel paste was used to place the electrodes. Before placing the electrodes, the surface of the skin was cleaned using a slightly abrasive NuPrep gel. Blood pressure was measured using the Kinetic Health Fully Automatic Blood Pressure Monitor (tested to European Society of Hypertension standards).

\section{Procedure}

All participants had been instructed to abstain from consuming any caffeinated beverage or indulging in extraneous exercise for about 2 hours prior to their scheduled session to ensure stable BP recordings. Upon arrival, they sat in a comfortable chair for about 15 minutes to allow time for their blood pressure to normalise. During this time, they were given the information sheet to remind them about the study and were requested to ask any questions or express any concerns they might have before providing written informed consent. They were then asked to indicate to what extent they were feeling certain positive 
and negative emotions at that moment using the 5-point Likert scale (1-very slightly or not at all to 5-extremely) of PANAS.

Before the experiment, all participants were given three practice trials using three neutral pictures (IAPS nos.7001, 7006, and 7183) and the startle probe was presented on two of the three pictures to familiarize them with the testing procedure. The eye blink component of the startle response was recorded as per published guidelines (Blumenthal et al., 2005). The affect-startle task was presented using E-prime 3.0 and the startle probe was presented simultaneously to both ears of the participants. The electromyography (EMG) recordings of the eye-blink startle reflex were made with two electrodes placed roughly $25 \mathrm{~mm}$ apart beneath the left eye over the orbicularis oculi muscle and one (reference) electrode placed on the left collar-bone. The EMG activity was recorded throughout the experiment at $1000 \mathrm{~Hz}$, with the bandwidth of the amplifier set to $25-500 \mathrm{~Hz}$. The recordings were made with participants sitting in a dimly lit sound-attenuated room.

For taking blood pressure measurements, participants were asked to sit in a heightadjustable chair with their back upright and feet lying flat on the ground. They were asked to sit with legs uncrossed, with their lower legs close together and at 90 degrees to their thighs. Blood pressure was measured from the dominant arm of the participants held up with support at the heart level. Three separate blood pressure measurements were made, allowing 1 minute before each successive reading.

Participants then took part in the startle experiment. The task instructions were presented to them on the screen (23" DELL desktop) and were also read out to them simultaneously. They were told that they will be shown some pleasant and unpleasant pictures and were asked to watch each picture attentively. They were asked to ignore the loud noise heard intermittently on the headphone. They were also instructed to remain as still as 
possible throughout the experiment. After the completion of the task the participants were debriefed about the study and reimbursed for their time and travel.

\section{Data Reduction}

The EMG signal was sampled at $1000 \mathrm{~Hz}$. The EMG startle data were rectified and smoothed (20-ms moving window average) and then scored off-line for response amplitude and latency values as in previous studies (Giakoumaki et al., 2010). A high-pass filter of 30 $\mathrm{Hz}$ and a low pass filter of $500 \mathrm{~Hz}$ were used. The onset latency window for the eye-blink reflex was set at 20-100 ms following the onset of the startle probe. The point of maximum amplitude (in $\mu \mathrm{V}$ ) within the $120 \mathrm{~ms}$ window following the startle probe onset was chosen as the peak amplitude. To determine the amplitude of the startle response, the average EMG for $50 \mathrm{~ms}$ prior to the onset latency window was subtracted from the peak amplitude. Data with startle equal to $0 \mu \mathrm{V}$ on any trial were coded as missing values $(<5 \%$; out of a total of 2124 trials presented (36 trials each for 59 participants), no startle response was noted for 21 trials of unpleasant, 21 trials of neutral, and 29 trials of pleasant stimuli). The latency to onset and peak were calculated as the time delay (in milliseconds) in the startle response initiation and the startle response reaching its maximum strength (i.e., peak amplitude), respectively in the 20-100 ms window following the onset of the startle probe. Following earlier similar studies (e.g., Corr \& Kumari, 2012; Kaviani et al, 2004), the data for all the participants was then checked for a difference of more than $95 \mathrm{~ms}$ between the onset and peak latencies or a shift of more than $50 \mu \mathrm{V}$ in the baseline amplitude and such trials were rejected.

\section{Data Analysis}

The latent BP model included all the three measurements of SBP and DBP as indicators. This model was tested using AMOS and after allowing the errors of SBP to 
correlate, the model yielded a very good fit to the data. The various indices of goodness of fit of structural equation model met the contemporary criteria of a good fit $\left[\chi^{2} / \mathrm{df}=0.482\right.$, GFI $=.984, \mathrm{AGFI}=.945, \mathrm{TLI}=1.04, \mathrm{CFI}=1.00, \mathrm{SRMR}=.0236$ and $\mathrm{RMSEA}=.000]$. The score of the latent BP factor was estimated using the data imputation feature of AMOS and based on this score the two BP groups (comparison and elevated) were created using the median split.

Independent samples $t$-tests were used to compare the comparison BP group and the elevated BP group on demographic, baseline mood and baseline startle measures, and $\chi^{2}$ test to compare them on gender distribution. Repeated-measures analyses of variance (ANOVAs) were used to compare the two groups on affective modulation of the startle amplitude and latencies to onset and peak with Valence (Pleasant, Neutral, Unpleasant) as the withinsubjects factor, and Group (comparison BP, elevated BP) as the between-subjects factor, followed by analysis of simple main effects and post-hoc pairwise comparisons (with LSD adjustment for multiple comparisons) where relevant. Initially, Gender was included as an additional between-group factor, but then removed because it showed no main or interactive effects. Since the comparison BP group was younger (see Results), we also examined possible associations between age and startle parameters using correlational and analyses of co-variance analyses but found no significant associations (all p- values $>.15$; not reported hereafter). Prior to running the above analyses, we examined data properties and found them to be suitable.

We also examined moderating effect of continuous moderator (latent BP) using repeated measures ANCOVA approach with valence as repeated measure and BP as covariate to examine if latent BP acted as a moderator of the relationship of Valence with startle response parameters (amplitude, latency to onset and peak). The significant interaction of covariate (latent BP) with the Valence was considered indicative of moderating effect and it 
was followed up by simple effect analysis (comparing the difference in startle response parameters across three valences for three BP levels (mean BP score and BP scores below and above one $\mathrm{SD}$ of the mean $\mathrm{BP}$ score). This comparison may be considered equivalent to the simple slope effects of the moderated regression analysis. In addition to this, a sub-sample analysis (comparing the correlation of $\mathrm{BP}$ with startle response parameters across the three valences) was also conducted for assessing the moderating effect of BP in the relationship. All analyses were conducted using Statistical Package for the Social Sciences (SPSS, version 23).

\section{Results}

\section{Sample Characteristics}

The comparison BP group was younger than the elevated BP group [ $\mathrm{t}(57)=2.22$, $\mathrm{p}=.03]$. The two groups had comparable gender distributions $\left[\chi^{2}(1)=1.51, \mathrm{p}=.22\right]$ (Table 1$)$. By design, the two groups differed in mean resting SBP [t $(57)=3.09, \mathrm{p}=.003]$ and DBP values [ $\mathrm{t}(57)=10.48, \mathrm{p}<.0001]$. The elevated BP group had $4.53 \mathrm{mmHg}$ higher SBP and $7.80 \mathrm{mmHg}$ higher DBP than the comparison group. Although the average SBP and DBP scores for both the comparison and elevated BP groups were in the lower range, they represented full normal BP variation range [ranging from 90/60 to 120/80; National Heart, Lung, and Blood Institute (NHLBI), n.d.] and $17 \%$ to $20 \%$ of the participants had their SBP and DBP, respectively, closer to the upper end of the normal range. The comparison and elevated BP groups did not differ on baseline affectivity, with no significant difference in their pre-experiment ratings of positive and negative affectivity assessed through PANAS [positive, $\mathrm{t}(57)=.848, \mathrm{p}=.40$; negative, $\mathrm{t}(57)=.618, \mathrm{p}=.54]$ (Table 1).

\section{Baseline Reactivity}


The two groups did not differ in startle amplitude to probes during the blank screens $[\mathrm{t}$ $(57)=.96, p=.34]$ (Table 2), baseline latency to onset $[\mathrm{t}(57)=.48, \mathrm{p}=.63]$ or baseline latency to peak $[\mathrm{t}(57)=.12, \mathrm{p}=.92]($ Table 3$)$.

\section{Affective Startle Modulation}

\section{Response Amplitude}

Repeated-measures ANOVA revealed a significant main effect of Valence $[\mathrm{F}(2,114)$ $\left.=11.69, \mathrm{p}<0.001, \eta^{2} p=.17\right]$ (Table 2), confirming significant startle attenuation by pleasant $(p=.001)$ and a trend for startle potentiation by unpleasant $(p=.091)$ pictures, both relative to neutral pictures. Startle amplitude during unpleasant pictures was significantly larger compared to that during the pleasant pictures $(\mathrm{p}<.001)$. There was no main effect of Group [F $\left.(1,57)=0.86, \mathrm{p}=0.36, \eta^{2} p=.02\right]$ but there was a significant Group $\mathrm{x}$ Valence interaction $[\mathrm{F}$ $\left.(2,114)=4.57, \mathrm{p}=0.012, \eta^{2} p=.07\right]$. Follow up analysis indicated a significant Valence effect $\left[\mathrm{F}(2,56)=10.38, \mathrm{p}=0.001, \eta^{2} p=.27\right]$, with significantly lower amplitude during pleasant $(p=.008)$ and higher amplitude during unpleasant $(p=.04)$ both compared to neutral picture viewing in the comparison BP group (Table 2, Figure 1). There was no significant effect of Valence in the elevated BP group $\left[\mathrm{F}(2,58)=1.58, \mathrm{p}=0.22, \eta^{2} p=.05\right]$ (Table 2, Figure 1).

Table 2: Descriptive statistics for startle amplitude in the comparison and elevated BP groups.

\begin{tabular}{cll}
\hline $\begin{array}{c}\text { Amplitude } \\
\text { (in microvolts) }\end{array}$ & $\begin{array}{l}\text { Comparison BP } \\
\text { group } \\
\text { Mean (SE) }\end{array}$ & $\begin{array}{l}\text { Elevated BP } \\
\text { group } \\
\text { Mean (SE) }\end{array}$ \\
\hline Baseline Reactivity & $28.01(7.52)$ & $19.45(4.88)$ \\
\hline
\end{tabular}


Affective Modulation

Pleasant $26.50(6.24) \quad 21.44(6.13)$

$\begin{array}{lll}\text { Neutral } & 31.58(6.68) & 22.94(6.57)\end{array}$

Unpleasant $\quad 34.49(6.39) \quad 23.23(6.28)$

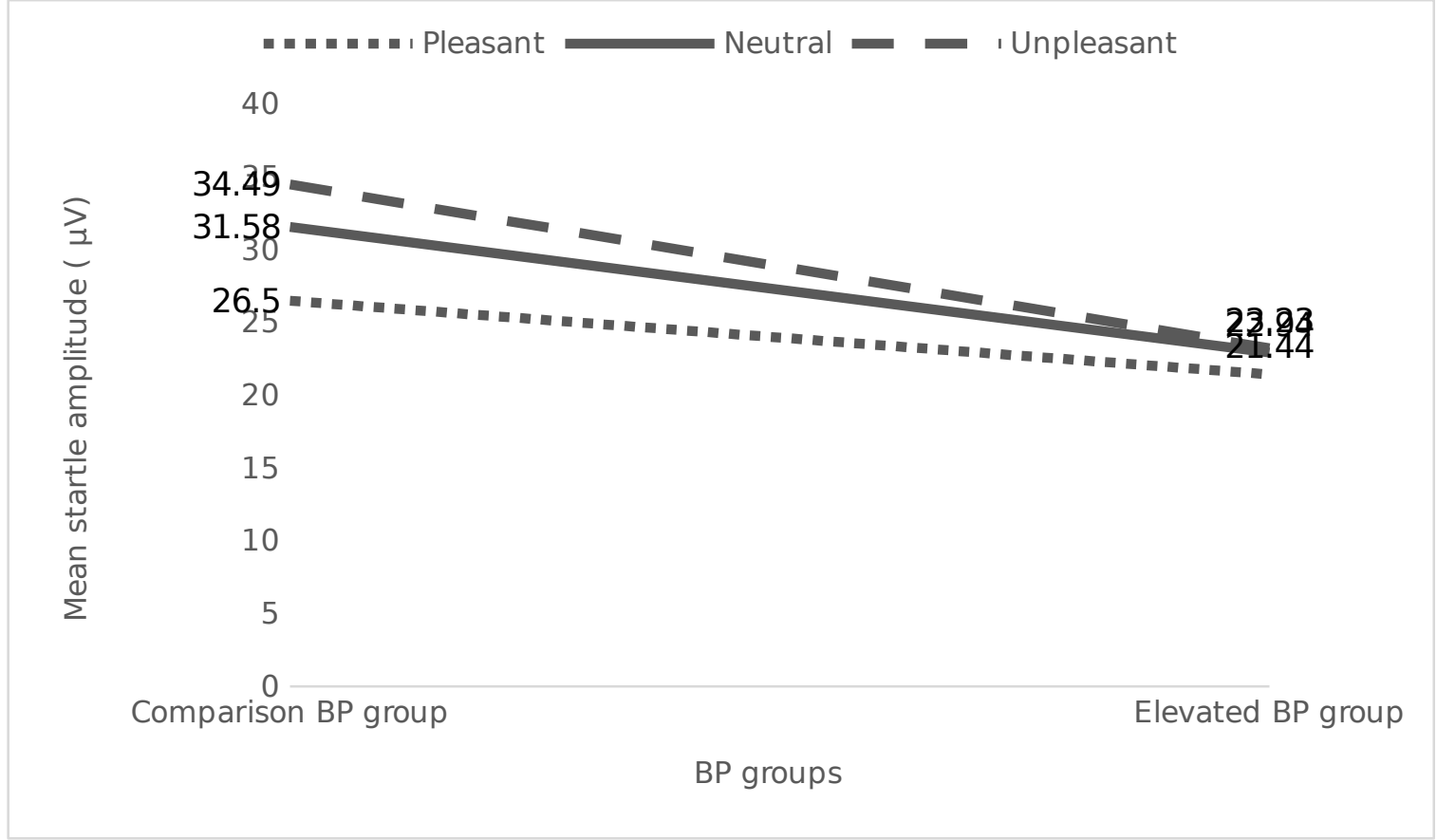

Figure 1: Mean startle amplitudes (in microvolts) for pleasant, neutral, and unpleasant picture categories in the comparison and elevated BP groups

\section{Latency to Response Onset}

The main effect of Valence was not significant $\left[\mathrm{F}(2,114)=2.14, \mathrm{p}=.12, \eta^{2} p=.04\right]$ but there was a significant Group $\mathrm{x}$ Valence interaction $\left[\mathrm{F}(2,114)=5.38, \mathrm{p}=.006, \eta^{2} p=.09\right]$ and a trend for the main effect of Group $\left[\mathrm{F}(1,57)=3.03, \mathrm{p}=.09, \eta^{2} p=.05\right]$ in the repeatedmeasures ANOVA output. Further analysis of this interaction indicated a significant valence 
effect $\left[\mathrm{F}(2,56)=4.86, \mathrm{p}=0.01, \eta^{2} p=.15\right]$ in the comparison BP group showing that the latencies during pleasant pictures were slower than neutral $(\mathrm{p}=0.005)$ and unpleasant pictures $(p=.08)$; the latencies for unpleasant and neutral pictures were not significantly different $(p=.23)$ (Table 3, Figure 2). The main effect of Valence was not significant in the elevated BP group $\left[\mathrm{F}(2,58)=2.39, \mathrm{p}=.10, \eta^{2} p=.08\right]$ (Table 3, Figure 2).

Table 3: Mean latencies to response onset and peak in the comparison and elevated BP groups

\begin{tabular}{lll}
\hline & $\begin{array}{l}\text { Comparison } \\
\text { BP group } \\
\text { Mean (SEM) }\end{array}$ & $\begin{array}{l}\text { Elevated BP group } \\
\text { Mean (SEM) }\end{array}$ \\
\hline \multicolumn{2}{l}{ Latency to response onset (in ms) } & \\
\hline Baseline & $23.58(.86)$ & $23.00(4.73)$ \\
\hline Affective Modulation & $25.94(1.00)$ & $22.37(0.98)$ \\
\hline Pleasant & $23.34(0.98)$ & $23.38(0.96)$ \\
Neutral & $24.29(0.89)$ & $21.73(0.87)$ \\
Unpleasant & $63.16(8.33)$ & $62.94(7.64)$ \\
\hline Latency to response peak (in ms) & \\
\hline Baseline & $66.58(1.56)$ & $63.77(1.53)$ \\
\hline Affective Modulation & $63.91(1.67)$ \\
\hline Pleasant & $66.38(1.70)$ & $63.12(1.49)$ \\
Neutral & $66.60(1.52)$ & \\
Unpleasant &
\end{tabular}




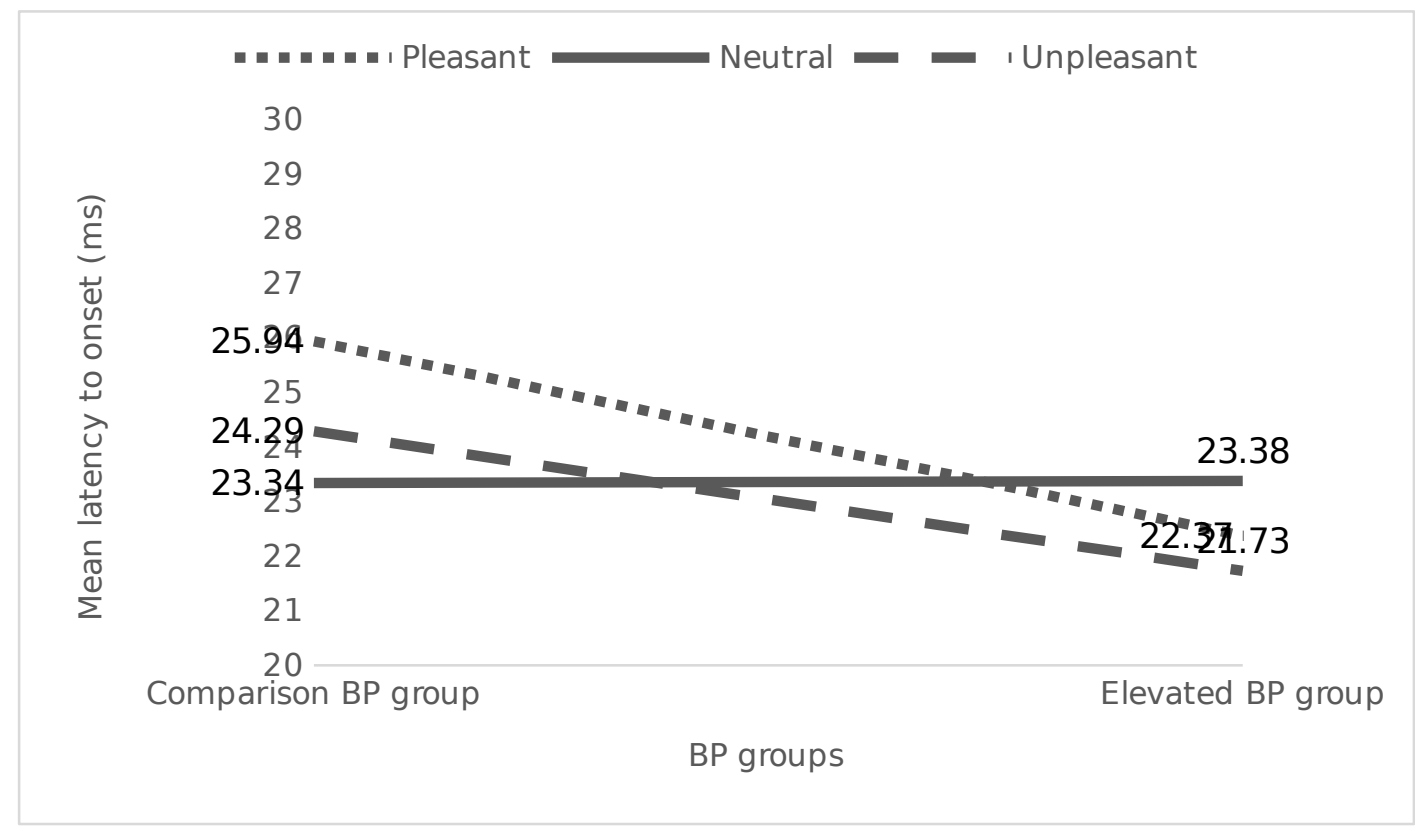

Figure 2: Mean latencies to response onset (in ms) for pleasant, neutral, and unpleasant valence in comparison and elevated BP groups

Latency to Response Peak

Repeated-measures ANOVA revealed that the main effects of Valence $[\mathrm{F}(2,114)=$. $\left.32, \mathrm{p}=.72, \eta^{2} p=.01\right]$ and Group $\left[\mathrm{F}(1,57)=1.52, \mathrm{p}=.22, \eta^{2} p=.03\right]$ were not significant. The Group $x$ Valence interaction was also non-significant $\left[\mathrm{F}(2,114)=1.18, \mathrm{p}=.31, \eta^{2} p=.02\right]$.

The aforesaid findings clearly document that the affective modulation of startle response is present in individuals in the comparison but not elevated BP group since the valence-specific potentiation/attenuation of startle amplitude was present in the comparison BP group but absent in the elevated BP group. The effect of valence was also not evident on the latency to startle onset in the elevated BP group whereas the comparison BP group showed a delayed latency to onset for positively-valanced compared to neutral stimuli.

Dichotomization of the latent BP variable (a continuous variable), however, may lead to loss of information and may yield false positive results (Altman \& Royston, 2006). Thus, we also tested the moderating effect of latent BP on the relationship of Valence with startle response parameters (amplitude, onset and peak of latency) using repeated-measures 
ANCOVA. Findings revealed a non-significant trend for the main effect of latent BP [F (1, $\left.57)=2.69, \mathrm{p}=.11, \eta^{2} p=.04\right]$ and almost significant Valence $\mathrm{x}$ latent BP interaction effect $[\mathrm{F}$ $\left.(2,114)=3.00, \mathrm{p}=.054, \eta^{2} p=.05\right]$ for startle amplitude.

The observed interaction effect of Latent BP X Valence was followed up by simple effects analysis in which the startle amplitude was compared across the three valence conditions for three values of the latent BP: Mean, Mean -1 SD, and Mean +1 SD. The findings revealed that at the mean value of latent BP (34.53) there was significant startle attenuation in the pleasant condition compared to neutral $(\mathrm{p}=.002)$ and unpleasant conditions $(p=.000)$. However, only marginally significant startle potentiation was noted in the unpleasant valence condition compared to neutral $(p=.097$; See Table 4, Figure 3). At the latent BP value of $1 \mathrm{SD}$ below the mean (32.12), significant startle attenuation in the pleasant condition and significant startle potentiation in the unpleasant condition were noted compared to neutral condition ( $\mathrm{p}=.002 \& .039$, respectively). The startle amplitude in the pleasant valence condition was also significantly lower than that in the unpleasant valence condition $(\mathrm{p}=.000)$. However, at the latent BP value of $1 \mathrm{SD}$ above the mean (36.93), the startle attenuation in the pleasant condition and potentiation in the unpleasant condition were found to be absent compared to neutral ( $\mathrm{p}=.179 \& .788$, respectively).

Table 4: Startle amplitude in the three valence conditions for three values of latent BP (Mean - 1 SD, Mean, Mean + 1 SD)

\begin{tabular}{cccc}
\hline \multirow{2}{*}{ Valence conditions } & \multicolumn{3}{c}{$\begin{array}{c}\text { Amplitude } \\
\text { (in microvolts) }\end{array}$} \\
\cline { 2 - 4 } & \multicolumn{3}{c}{ Latent BP } \\
\cline { 2 - 4 } Pleasant & Mean - 1 SD & Mean & Mean + 1 SD \\
\hline \multirow{2}{*}{ Ple } & $29.90(6.13)$ & $23.93(4.31)$ & $17.95(6.13)$
\end{tabular}


Neutral

$34.51(6.56)$

Unpleasant

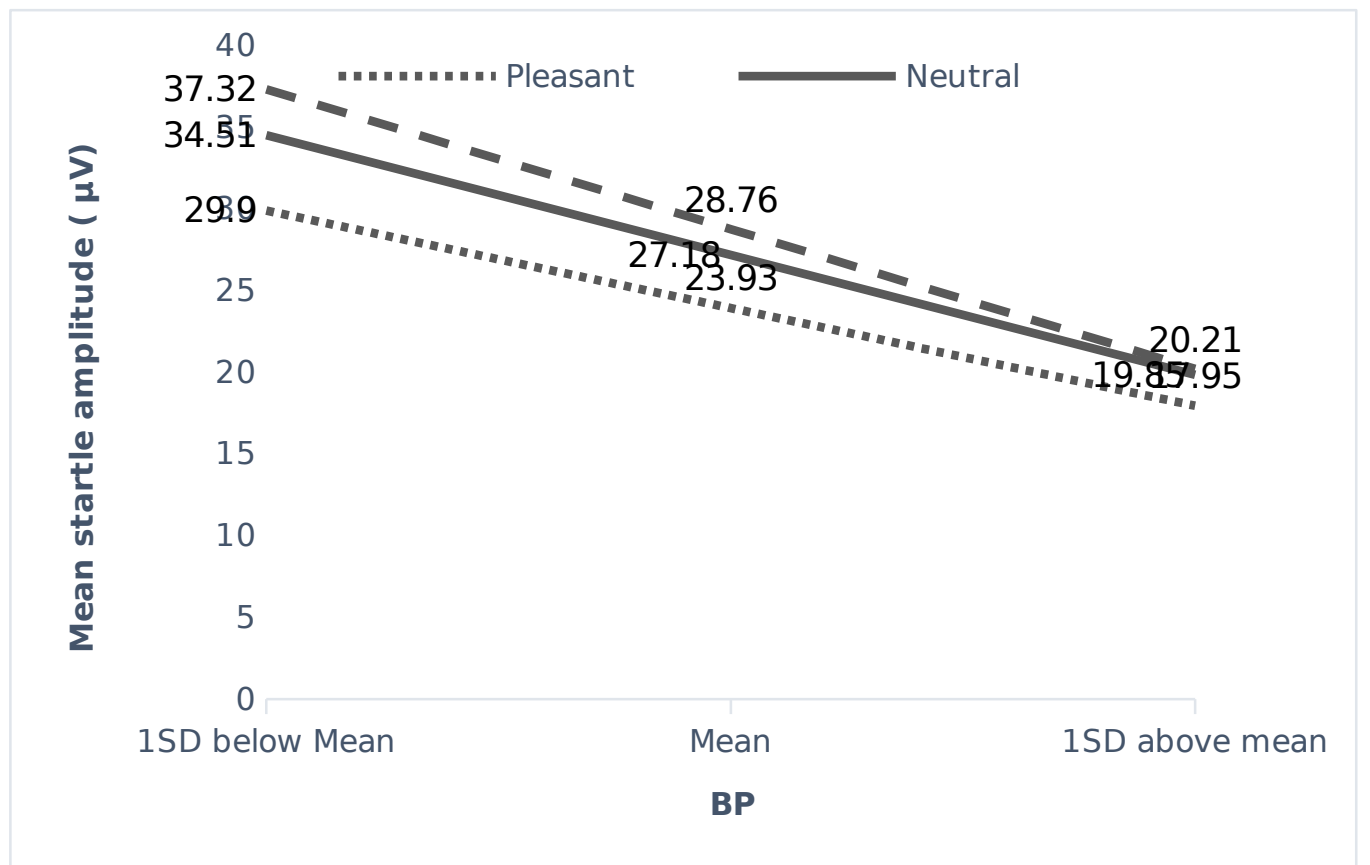

Figure 3: Moderating role of latent BP in the relationship of valence and startle amplitude

The aforesaid observations of ANCOVA are similar to the findings obtained with the dichotomised latent BP group ANOVA, since significant valence-specific modulation of startle was noted at latent BP 1 SD below the mean (similar to the findings obtained for the comparison BP group) and the valence-specific startle modulation was absent at $+1 \mathrm{SD}$ of latent BP (similar to the findings obtained for the elevated BP group). As far as the effect size of this interaction effect and the post-hoc observed power is concerned, the findings revealed that the observed effect size and power were relatively higher in the categorical approach (.074 \& .766, respectively) as compared to that of ANCOVA (.050 \& .571, respectively) where latent $\mathrm{BP}$ was used as continuous variable. 
Findings of sub-sample analysis for startle amplitude revealed that in the comparison BP group, latent BP showed marginally significant correlations with startle amplitude in the pleasant $(\mathrm{r}=-.344, \mathrm{p}=.067)$, neutral $(\mathrm{r}=-.343, \mathrm{p}=.069)$, and unpleasant $(\mathrm{r}=-.360, \mathrm{p}=.055)$ valence conditions. However, the same was not true for the elevated BP group which showed non-significant correlations with startle amplitude in all the three valence conditions, i.e., pleasant $(r=-.096, p=.615)$, neutral $(r=-.053, p=.779)$, and unpleasant $(r=-.062, p=.744)$. These findings provide further support for the moderating role of BP in the valence-startle amplitude relationship and suggest that affect related modulation of startle amplitude is present for lower levels of BP but the same is not observed when BP is elevated or starts increasing even in the normal range.

Similar repeated measures ANCOVA was done for the latency to onset of startle response and findings revealed a non-significant main effect of latent $\mathrm{BP}[\mathrm{F}(1,57)=.79$, $\left.\mathrm{p}=.38, \eta^{2} p=.01\right]$ as well as interaction of Valence $\mathrm{x}$ latent $\mathrm{BP}\left[\mathrm{F}(2,114)=.92, \mathrm{p}=.40, \eta^{2} p\right.$ $=.02]$. Unlike the categorical approach where BP level-specific valence effect on latency to onset of startle response was noted, the findings of the present analysis failed to provide support for this. The findings obtained from the sub-sample analysis also showed nonsignificant correlations of latent BP with latency to startle onset, both for the comparison and the elevated BP groups, in the pleasant $(r=.200, p=.299 \& \mathrm{r}=.225, \mathrm{p}=.233$, respectively), neutral $(\mathrm{r}=.092, \mathrm{p}=.637 \& \mathrm{r}=-.122, \mathrm{p}=.519$, respectively), and unpleasant $(\mathrm{r}=.285, \mathrm{p}=.134 \&$ $\mathrm{r}=.040, \mathrm{p}=.835$, respectively) valence conditions.

The repeated measures ANCOVA for the latency to peak yielded a similar nonsignificant main effect of latent $\mathrm{BP}\left[\mathrm{F}(1,57)=.29, \mathrm{p}=.60, \eta^{2} p=.005\right]$ as well as the interaction of Valence and latent BP $\left[\mathrm{F}(2,114)=.80, \mathrm{p}=.45, \eta^{2} p=.014\right]$. This finding is in line with the findings obtained for the latency to peak of startle response using the categorical BP variable. The sub-sample analysis for the latency to peak revealed non-significant 
correlations of latent BP with the three valence categories in both the comparison [pleasant $(\mathrm{r}=.076, \mathrm{p}=.695)$, neutral $(\mathrm{r}=.069, \mathrm{p}=.724$ and unpleasant $(\mathrm{r}=.104, \mathrm{p}=.593)]$ and elevated $\mathrm{BP}$ [pleasant $(\mathrm{r}=.140, \mathrm{p}=.461)$, neutral $(\mathrm{r}=.130, \mathrm{p}=.492$ and unpleasant $(\mathrm{r}=.116, \mathrm{p}=.542)$ ] groups.

\section{Discussion}

The study was designed to investigate reduced emotional responsiveness (or emotional dampening) in response to elevated BP using the well-established affect modulated eye-blink startle paradigm. Consistent with numerous previous findings (Lang, Bradley, \& Cuthbert, 1990), we observed a linear modulation of startle response (i.e. startle attenuation for positive and potentiation for negative stimuli) across the whole sample. Importantly, this ASM effect was significantly present only in the comparison BP group, and absent in the elevated BP group, consistent with our a priori hypothesis (that even marginal elevations in $\mathrm{BP}$ in the normal range may be associated with reduced emotional reactivity) and previous findings of impaired emotion recognition in elevated BP. This finding obtained using categorical BP variable was further substantiated by similar findings when BP was explored as a continuous moderating variable in the relationship of valence and startle amplitude. The findings of the sub-sample analysis (i.e., marginally significant correlations of BP with startle amplitude in comparison but not elevated BP group) provide further evidence for the lack of ASM with elevation in BP. The observed consistency in the findings across different analytic approach suggests that reduced emotional reactivity (emotional dampening), at involuntary level, appears to be a robust correlate of elevated BP. It also implies that the affect-startle paradigm may be used to gauge the reduced emotional reactivity in relation to elevated BP (at least in terms of startle amplitude). Our findings that BP does not affect the time taken for the involuntary startle response to reach its peak amplitude was also found to be consistent across 
different statistical analyses suggesting the latency to peak of the startle response is not a sensitive parameter to tap the reduced emotional reactivity in relation to elevated BP.

It is, however, important to note here that the evidence for reduced emotional reactivity using affect-startle paradigm was stronger for startle amplitude but not for other parameters of startle response (viz., latency to onset and peak of startle response). This lack of ASM in the elevated BP group was evident in both amplitude and latency of the onset of startle response when comparison was made by dichotomizing the latent BP score (comparison and elevated BP group). However, this finding (reduced ASM with elevation in BP) was replicated only for affect startle (and not for latency of the onset of startle response) when latent BP was used as a continuous moderator variable in ANCOVA. Thus. it would be premature to conclude that emotional dampening in response to elevated $\mathrm{BP}$ is also observed in terms of onset latency despite the fact that we noted such an effect when participants were categorized into comparison and elevated BP groups. As researchers have noted that categorization of continuous variable may lead to false positive findings (Altman \& Royston, 2006), the likelihood of the same cannot be denied in the case of observed dampened emotional reactivity in elevated BP group in terms of latency of the onset of startle response. Thus, future research is needed to explore how far the observed dampening in terms of latency to the onset of startle response is a characteristic correlate of elevated BP (and is not a statistical artefact) using a relatively larger sample and treating BP as a continuous measure.

Our findings showing lack of significant ASM (at least in terms of startle amplitude) add to the existing literature and indicate that the emotional reactivity aspect of emotional responsiveness (that was neglected in earlier studies) is also associated with BP variations in the normal range. It is well-known that emotional arousal increases BP (Bedi, Varshney, \& Babbar, 2000) and it is plausible that such people may consciously or unconsciously develop blunted emotional responding as an adaptive mechanism to down-regulate the experience of 
intense emotions, thereby preventing further elevation in BP. The findings not only confirm our a priori hypothesis that elevated BP in the normal range would be associated with reduced ASM but also support the notion that emotional dampening in association with elevated BP occurs at an involuntary stage of information processing. An alternative explanation for the findings could be offered based on the generally observed higher negative affectivity in individuals with elevated BP (e.g., Ong \& Allaire, 2005). Implicit priming towards negative emotion may counteract the effect of positive emotions on startle response and, at the same time, may not further potentiate the effect of negative emotional stimuli because of already existing higher levels of negative affectivity. However, this implicit priming hypothesis seems a less probable explanation for the present study since baseline startle reactivity was comparable in the two groups and the groups did not differ in preexperiment positive or negative affectivity. This possibility may be tested conclusively by comparing normotensives with hypertensives (or comparing normotensives with low and high BP elevations), with relatively higher level of negative affect in the latter group.

Studies involving patients with amygdala lesions (Buchanan, Tranel, \& Adolphs, 2004; Kettle, Andrewes, \& Allen, 2006) have reported an overall attenuated startle reflex magnitude, providing evidence for the role of amygdala in normal startle reactivity. Although the present study did not allow for the direct measurement of this possibility, there are studies (e.g., Saha, 2005) indicating the role of the central nucleus of amygdala (CeA) in blood pressure regulation, particularly in response to unpleasant affective stimuli. Further empirical investigation is required to establish the mediating role of the amygdala between elevated BP and attenuated startle reactivity. Another mechanism involved in reduced ASM in the elevated BP group may be the baroreceptor mechanism implicated in the BP-emotional dampening relationship (McCubbin et al, 2011; McCubbin et al, 2014). It has long been believed that baroreceptor mechanism is responsible for just the short-term regulation of BP 
and has no role in regulating BP chronically (Kougias, Weakley, Yao, Lin, \& Chen, 2010). However, recent studies have shown that the baroreflex system may continue to adjust changes in BP for several weeks without resetting to a new set point (e.g., Joyner, Charkoudian, \& Wallin, 2008). Moreover, activation of the baroreflex mechanism has been shown to be linked with attenuated response to auditory startle stimuli (Nyklícek, Wijnen, \& Rau, 2005). Although we did not find a significantly lower baseline startle amplitude in the elevated BP group, compared to the comparison BP group, the data hinted towards this effect (Table 2, Figure 1). Thus, the baoreceptor mechanism may play a mediating role in the BPemotional dampening relationship.

The reduced ASM in the elevated BP group is similar to those seen in depression (Dichter \& Tomarken, 2008) and anhedonia (Allen, Trinder, \& Brennan, 1999). A suspected link between elevated BP and depression may be because of increased adrenergic activity in depressive state which has a pressor effect upon the cardiovascular system (Siever \& Davis, 1985). Furthermore, depression is one of the common comorbid conditions associated with cardiovascular disorders (AlKhathami, et al., 2017) and depression has also been linked with blunted response to emotional stimuli (e.g. Kaviani et al., 2004). Thus, the present finding of reduced emotional reactivity in the elevated BP group may have significant clinical implications for understanding the comorbidity of depression and cardiovascular disorders. The present data and design though do not allow to directly test and support this speculation (as comparison and elevated BP groups did not differ in negative affect), it needs to be tested in future studies involving individuals with clinical hypertension, and comorbid anxiety, depression or other negative affective states.

The findings of the present study are encouraging and support the notion that elevated $\mathrm{BP}$ is associated with reduced emotional reactivity, but their generalizability is limited because of several possible limitations. First, the sample consisted of participants who 
volunteered themselves. Thus, the present sample is likely to suffer from self-selection bias and may not be representative of the normotensive population. Second, some of the stimuli in the present study differed based on participant gender and thus may generate complexities in interpreting the results, particularly when the primary independent variable (affect-modulated startle amplitude) does show sex differences (e.g., Bradley, Codispoti, Sabatinelli, \& Lang, 2001). Thus, it is recommended that future studies address this issue by selecting the same set of stimuli for both males and females (rather than using the images with large expected gender differences). Such an attempt will allow to compare the differences in findings across studies that use gender-specific set of stimuli and those using the same set of stimuli irrespective of the gender of the participants. Third, the validity of the findings might have been strengthened had other electrophysiological measures of involuntary emotional reactivity [e.g. galvanic skin response, respiration rate, facial EMG of muscles involved in emotions, event related potentials (ERPs)] been obtained along with startle reflex. The use of ERP paradigms to study emotional responding (e.g. with N1, P2, P3 indices) may also help to establish whether the effects we observed (i.e. reduced emotional reactivity in the elevated BP group indexed with startle probes that were delivered 2-5 s after picture onset) were related to an aberrant responding at an earlier stage of information processing. Similarly, future research may also focus on uncovering other aspects of implicit emotion processing difficulties in relation to elevated BP using stochastic diffusion models (Ratcliff, 1978). The diffusion model allows to analyse the speed of information uptake, amount of information used in decision making, possible decision biases and duration of non-decisional processes (see Voss, Nagler, \& Lerche, 2013 for details). Thus, use of diffusion model may help to assess whether elevated BP linked deficit in emotional processing occurs at level of decision making or at other early stages of processing of information intake. 
Future research is also needed to examine whether these findings generalise to elevated BP in the hypertensive range. Further studies also need to use within-subjects designs to examine this association of reduced ASM in elevated BP conditions. For example, studies could use pre-post assessments of ASM to assess effects of experimental manipulations aimed at lowering BP on levels of ASM. If lowered BP shows return to normal ASM, ASM can serve as a biomarker of emotion linked baroreceptor responses (facilitation and inhibition) implicated in hypertension and may have implications for identifying the underlying brain mechanism of baroreceptor modulation (Nosaka, 1996). Future investigations can also incorporate self-report measures of such affective disorders as anxiety and depression to gain better insight into the present findings.

\section{Conclusion}

The findings of the present study demonstrate reduced ASM of startle amplitude in individuals with even marginally elevated $\mathrm{BP}$ within the normal range of $\mathrm{BP}$ variations and extend the emotional dampening hypothesis to include reduced emotional reactivity as indexed with ASM. While some evidence was also obtained for altered ASM in the latency to onset of startle response, it would be premature to make any conclusion in this regard till future investigations establish its salience.

\section{Declarations of interest: None}

Ethics approval: The study has been approved by the King's College Research Ethics Committee (Ref: HR-16/17-3826) and has therefore been performed in accordance with the ethical standards laid down in the 1964 Declaration of Helsinki. 
Disclosure: We hereby disclose that we have reported at appropriate places how we determined the sample size, all the data exclusions (if any), the conditions of the experiment, and all the measures used in the present study.

\section{References}

AlKhathami, A. D., Alamin, M. A., Alqahtani, A. M., Alsaeed, W. Y., AlKhathami, M. A., \& Al-Dhafeeri, A. H. (2017). Depression and anxiety among hypertensive and diabetic primary health care patients: Could patients' perception of their diseases control be used as a screening tool?. Saudi Medical Journal, 38(6), 621-628.

Allen, N. B., Trinder, J., \& Brennan, C. (1999). Affective startle modulation in clinical depression: preliminary findings. Biological Psychiatry, 46(4), 542-550.

Altman, D. G., \& Royston, P. (2006). The cost of dichotomising continuous variables. $B M J$, $332(7549), 1080$.

Bedi, M, Varshney, V.P., \& Babbar, R. (2000). Role of Cardiovascular Reactivity to Mental Stress in Predicting Future Hypertension. Clinical and Experimental Hypertension, 22 (1), 1-22.

Blumenthal, T. D., Cuthbert, B. N., Filion, D. L., Hackley, S., Lipp, O. V., \& Van Boxtel, A. (2005). Committee report: Guidelines for human startle eyeblink electromyographic studies. Psychophysiology, 42(1), 1-15.

Bradley, M. M., Codispoti, M., Sabatinelli, D., \& Lang, P. J. (2001). Emotion and motivation II: Sex differences in picture processing. Emotion, 1, 300 -319.

Buchanan, T. W., Tranel, D., \& Adolphs, R. (2004). Anteromedial temporal lobe damage blocks startle modulation by fear and disgust. Behavioral Neuroscience, 118(2), 429-437. 
Cohen, J. (1969). Statistical power analysis for the behavioural sciences. New York: Academic Press.

Corr, P. J., \& Kumari, V. (2013). Effect of D-amphetamine on emotion-potentiated startle in healthy humans: implications for psychopathy and antisocial behaviour. Psychopharmacology, 225(2), 373-379.

Corr, P.J., Wilson, G.D., Fotiadou, M., Kumari, V., Gray, N.S., Checkley, S.A., Gray, J.A. (1995). Personality and affective modulation of the startle reflex. Personality and Individual Differences, 19, 543-553.

Dichter, G. S., \& Tomarken, A. J. (2008). The chronometry of affective startle modulation in unipolar depression. Journal of Abnormal Psychology, 117(1), 1-15.

Forouzanfar, M. H., Liu, P., Roth, G. A., Ng, M., Biryukov, S., Marczak, L., ... \& Ali, R. (2017). Global burden of hypertension and systolic blood pressure of at least 110 to 115 mm Hg, 1990-2015. JAMA, 317(2), 165-182.

Giakoumaki, S. G., Bitsios, P., Frangou, S., Roussos, P., Aasen, I., Galea, A., \& Kumari, V. (2010). Low baseline startle and deficient affective startle modulation in remitted bipolar disorder patients and their unaffected siblings. Psychophysiology, 47(4), 659-668.

Grillon, C., \& Baas, J. (2003). A review of the modulation of the startle reflex by affective states and its application in psychiatry. Clinical Neurophysiology, 114(9), 1557-1579.

Jain, D., Shukla, M., \& Pandey, R. (2017). Blood pressure variations and emotional dampening: preliminary evidence for the curvilinear relationship. SIS Journal of Projective Psychology \& Mental Health, 24 (1), 18-25.

Jonas, B. S., \& Lando, J. F. (2000). Negative affect as a prospective risk factor for hypertension. Psychosomatic Medicine, 62(2), 188-196. 
Joyner, M. J., Charkoudian, N., \& Wallin, B. G. (2008). A sympathetic view of the sympathetic nervous system and human blood pressure regulation. Experimental Physiology, 93(6), 715-724.

Kaviani, H., Gray, J.A., Checkley, S.A., Raven, P.W., Wilson, G.D., \& Kumari, V. (2004). Affective modulation of the startle response in depression: Influence of the severity of depression, anhedonia and anxiety. Journal of Affective Disorders, 83(1), 21-31.

Kettle, J. W., Andrewes, D. G., \& Allen, N. B. (2006). Lateralization of the startle reflex circuit in humans: An examination with monaural probes following unilateral temporal lobe resection. Behavioral Neuroscience, 120(1), 24-39.

Kougias, P., Weakley, S. M., Yao, Q., Lin, P. H., \& Chen, C. (2010). Arterial baroreceptors in the management of systemic hypertension. Medical Science Monitor: International Medical Journal of Experimental and Clinical Research, 16(1), RA1-8.

Kumari, V., Corr, P.J., Wilson, G.D., Kaviani, H., Thornton, J.C., Checkley, S.A., Gray, J.A. (1996). Personality and modulation of the startle reflex by emotionally-toned film-clips. Personality and Individual Differences, 21, 1029-1041.

Lang, P. J., Bradley, M. M., \& Cuthbert, B. N. (2005). International Affective Picture System (IAPS): Affective Ratings of Pictures and Instruction Manual. Technical Report A-6, Gainesville, FL: University of Florida.

Lang, P. J., Bradley, M. M., \& Cuthbert, B. N. (1990). Emotion, attention, and the startle reflex. Psychological Review, 97(3), 377-395.

Lang, P. J., \& McTeague, L. M. (2009). The anxiety disorder spectrum: Fear imagery, physiological reactivity, and differential diagnosis *. Anxiety, Stress, \& Coping, 22(1), 525. 
Markovitz, J. H., Matthews, K. A., Whooley, M., Lewis, C. E., \& Greenlund, K. J. (2004). Increases in job strain are associated with incident hypertension in the CARDIA Study. Annals of Behavioral Medicine, 28(1), 4-9.

McCubbin, J. A., Loveless, J. P., Graham, J. G., Hall, G. A., Bart, R. M., Moore, D. D., ... \& Thayer, J. F. (2014). Emotional dampening in persons with elevated blood pressure: affect dysregulation and risk for hypertension. Annals of Behavioral Medicine, 47(1), 111-119.

McCubbin, J. A., Merritt, M. M., Sollers III, J. J., Evans, M. K., Zonderman, A. B., Lane, R. D., \& Thayer, J. F. (2011). Cardiovascular emotional dampening: The relationship between blood pressure and recognition of emotion. Psychosomatic Medicine, 73(9), 743-750.

Mayor, S. (2016). Non-communicable diseases now cause two thirds of deaths worldwide. BMJ: British Medical Journal (Online), 355.

National Heart, Lung, and Blood Institute. (n.d.). Hypotension. Retrieved from https://www.nhlbi.nih.gov/health-topics/hypotension

Nosaka, S. (1996). Modifications of arterial baroreflexes: obligatory roles in cardiovascular regulation in stress and poststress recovery. The Japanese Journal of Physiology, 46(4), 271-288.

Nyklíček, I., Wijnen, V., \& Rau, H. (2005). Effects of baroreceptor stimulation and opioids on the auditory startle reflex. Psychophysiology, 42(2), 213-222.

Ong, A. D., \& Allaire, J. C. (2005). Cardiovascular intraindividual variability in later life: the influence of social connectedness and positive emotions. Psychology and Aging, 20(3), 476-485. 
Pei, D., Chen, Y. L., Tang, S. H., Wu, C. Z., Lin, J. D., Chang, Y. L., ... \& Wang, J. Y. (2011). Relationship of blood pressure and cardiovascular disease risk factors in normotensive middle-aged men. Medicine, 90(5), 344-349.

Pury, C. L., McCubbin, J. A., Helfer, S. G., Galloway, C., \& McMullen, L. J. (2004). Elevated resting blood pressure and dampened emotional response. Psychosomatic Medicine, 66(4), 583-587.

Rapsomaniki, E., Timmis, A., George, J., Pujades-Rodriguez, M., Shah, A. D., Denaxas, S., ... \& Williams, B. (2014). Blood pressure and incidence of twelve cardiovascular diseases: lifetime risks, healthy life-years lost, and age-specific associations in 1.25 million people. The Lancet, 383(9932), 1899-1911.

Ratcliff, R. (1978). A theory of memory retrieval. Psychological Review, 85(2), 59-108.

Ray, W. J., Molnar, C., Aikins, D., Yamasaki, A., Newman, M. G., Castonguay, L., \& Borkovec, T. D. (2009). Startle response in generalized anxiety disorder. Depression and Anxiety, 26(2), 147-154.

Saha, S. (2005). Role of the central nucleus of the amygdala in the control of blood pressure: descending pathways to medullary cardiovascular nuclei. Clinical and Experimental Pharmacology and Physiology, 32(5-6), 450-456.

Shukla, M., Gupta, S., \& Pandey, R. (2017). Behavioural Manifestation and Awareness of Emotional Processing Deficits in Individuals with Hypertension: Evidence for Emotional Dampening Hypothesis. Indian Journal of Clinical Psychology, 149(2), 107-117.

Shukla, M., Pandey, R., Jain, D., \& Lau, J. Y. (2018). Poor emotional responsiveness in clinical hypertension: Reduced accuracy in the labelling and matching of emotional faces amongst individuals with hypertension and prehypertension. Psychology \& Health, 33(6), 765-782. 
Shukla, M., Pandey, R., \& Lau, J. Y. (2019). Assessing emotional processing difficulties in normotensive individuals with high and isolated blood pressure elevations. International Journal of Psychology, 54(2), 214-222.

Siever, L. J., \& Davis, K. L. (1985). Overview: Toward a dysregulation hypothesis of depression. The American Journal of Psychiatry, 142(9), 1017-1031.

Taylor-Clift, A., Morris, B. H., Rottenberg, J., \& Kovacs, M. (2011). Emotion-modulated startle in anxiety disorders is blunted by co-morbid depressive episodes. Psychological Medicine, 41(1), 129-139.

Voss, A., Nagler, M., \& Lerche, V. (2013). Diffusion models in experimental psychology. Experimental Psychology, 60, 385-402.

Vrana, S. R., Spence, E. L., \& Lang, P. J. (1988). The startle probe response: a new measure of emotion?. Journal of Abnormal Psychology, 97(4), 487-491.

Watson, D., Clark, L. A., \& Tellegen, A. (1988). Development and validation of brief measures of positive and negative affect: the PANAS scales. Journal of Personality and Social Psychology, 54(6), 1063-1070. 